\title{
Polarimetric calibration of a spectro- polarimeter for remote sensing and characterization of aerosols
}

Martijn Smit, Jeroen Rietjens, Jochen Campo, Joost aan de Brugh, Aaldert van Amerongen, et al.

Martijn Smit, Jeroen Rietjens, Jochen Campo, Joost aan de Brugh, Aaldert van Amerongen, Otto Hasekamp, Jochen Landgraf, Xin Liu, "Polarimetric calibration of a spectro-polarimeter for remote sensing and characterization of aerosols," Proc. SPIE 11852, International Conference on Space Optics ICSO 2020, 1185232 (11 June 2021); doi: 10.1117/12.2599475

SPIE Event: International Conference on Space Optics - ICSO 2021, 2021, Online Only 


\section{International Conference on Space Optics-ICSO 2020}

Virtual Conference

30 March-2 April 2021

Edited by Bruno Cugny, Zoran Sodnik, and Nikos Karafolas
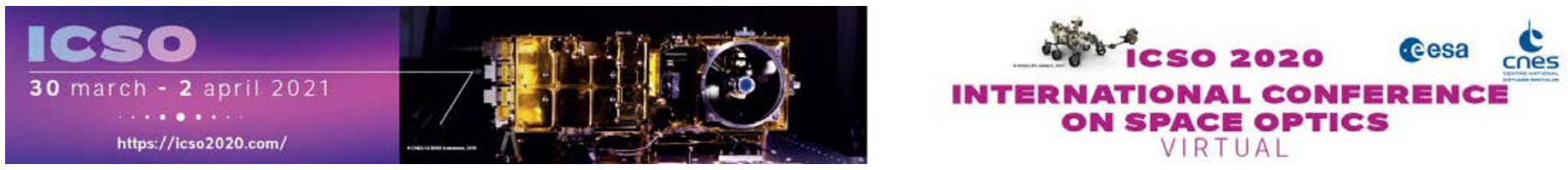

\section{Polarimetric calibration of a spectro-polarimeter for remote sensing and characterization of aerosols}

\section{Cesa isoporeseatings denes}




\title{
Polarimetric calibration of a spectro-polarimeter for remote sensing and characterization of aerosols
}

\author{
Martijn Smit ${ }^{\mathrm{a}}$, Jeroen Rietjens ${ }^{\mathrm{a}}$, Jochen Campo ${ }^{\mathrm{a}}$, Joost aan de Brugh ${ }^{\mathrm{a}}$, Aaldert van Amerongen ${ }^{\mathrm{a}}$, \\ Otto Hasekamp ${ }^{\mathrm{a}}$, Jochen Landgraf ${ }^{\mathrm{a}}$ and Xin Liu ${ }^{\mathrm{a}, \mathrm{b}}$

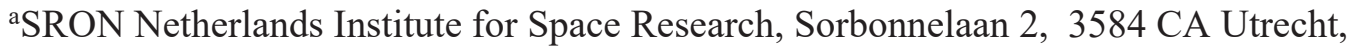 \\ the Netherlands \\ ${ }^{\mathrm{b} S c h o o l ~ o f ~ o p t i c s ~ a n d ~ p h o t o n i c s, ~ B e i j i n g ~ I n s t i t u t e ~ o f ~ T e c h n o l o g y, ~ H a i d i a n ~ D i s t r i c t, ~ B e i j i n g, ~ C h i n a ~}$
}

High accuracy multi-angle polarimetry is of crucial importance for remote sensing of aerosol properties with accuracies demanded by climate and air quality studies. In this contribution, we discuss the polarimetric calibration of the multi-angle polarimeter "SPEX airborne". SPEX airborne is a multi-angle viewing instrument providing snapshot measurements of spectral radiance and degree of linear polarization at fixed viewing angles. Radiance and polarization are measured as a continuous function of wavelength in the 400-760nm range, at nine viewing angles equally distributed over an angular range of $-56^{\circ}$ to $+56^{\circ}$. Each viewing-angle aperture has a swath of $7^{\circ}$ with an instantaneous field of view of $0.5^{\circ} \times 1^{\circ}(\mathrm{cross}-$ times along-track).

SPEX airborne measures the degree and angle of linear polarization of scattered sunlight by means of spectral modulation. For each field of view, the instrument records two modulated spectra. Ideally, these are perfectly in anti-phase, such that the sum of the modulated spectra is modulation free and gives the spectral radiance. The state of linear polarization is derived from the scaled difference of the modulated spectra. As a result of finite image quality at the focal plane, any spectropolarimeter using spectral modulation will show different polarimetric responses for the two modulated spectra, which breaks the anti-phase symmetry. For SPEX airborne this is indeed the case, and special care is taken both in the calibration and in the data processing. Ignoring this can lead to errors both in polarimetric and radiometric measurements. It is shown however that these errors are quite small.

In this contribution, we will outline the spectral modulation technique employed by SPEX airborne to measure the state of linear polarization, explain which instrumental parameters are to be determined by calibration and how they feature in the data processing chain. We discuss the polarization calibration setup and how polarization calibration measurements are processed into useful calibration data. Taking a Mueller matrix approach, we show how differences in polarimetric responses can be mitigated, while also other imperfections like telescope polarization are accounted for without extra calibration effort. We also present a scheme to correct for sharp features in the solar spectrum, which would otherwise mix into the modulation spectra via the finite slit-width of the spectrometer and result in polarimetric errors. The effect of telescope polarization is also discussed

Polarimetric calibration of SPEX airborne is relevant for SPEXone, a compact multi-angle polarimeter that builds on SPEX airborne heritage and space-borne spectrometer heritage within the Netherlands. SPEXone has been developed for NASA's PACE mission, which has a planned launch date in 2023.

Keywords: spectro-polarimetry, multi-angle, airborne, remote sensing, aerosols

\section{INTRODUCTION}

It is a well-established fact that aerosol impact our climate and air-quality significantly. The present situation is that the overall cooling effect due to aerosol may be as large as the heating effect of the strongest greenhouse gas. However, large uncertainties in aerosol-cloud interactions hamper reliable climate forecasts. These uncertainties must urgently be reduced, by improving observations of aerosol on a global scale, and by extending the parameter space of measured particle properties. Aerosol (and cloud) properties can only be unambiguously determined from space-based instruments that measure both intensity and polarization at multiple viewing angles and multiple wavelength with a wide swath to enable global coverage at daily timescales [1]-[5]. The present-day standard for remote sensing multi-angle polarimetric instrumentation for atmospheric aerosol characterization is to deliver polarimetric measurements with an error on the degree of linear polarization not larger than a few times 0.001 . The realization of SPEX airborne is part of a development in The Netherlands towards several possible realizations of space-based, SPEX-like instruments [13]. The original optical module of SPEX airborne was designed and built by a consortium of Dutch knowledge institutes and space technology 
companies [6][7]. Preliminary characterization \& performance tests under laboratory conditions demonstrated excellent performance [8]-[12], indicating that the instrument concept would be capable to meet stringent performance requirements for aerosol characterization of the Earth's atmosphere. SPEX airborne was subsequently developed by SRON as a payload for NASA's high altitude atmospheric research aircraft ER-2 [18]. In October 2020 the instrument participated in a flight campaign that is part of SCARBO (Space CARBon Observatory) [19], a study funded by the European Commission to develop a new miniature CO2 sensor "NanoCarb" [20] to be deployed together with SPEX-like instrument on a future constellation of satellites.

The first space-borne derivative of SPEX airborne is SPEXone, a compact, $6 \mathrm{dm}^{3}$-sized spectropolarimeter with five fixed viewing angles, developed for NASA's Phytoplankton, Aerosols, Clouds and ocean Ecosystems (PACE) mission which is scheduled for launch in 2023. For SPEXone the requirement on the DoLP error is $<0.003$. Details of SPEXone, including optical and mechanical design, integration, test \& verification approach are provided in [14],[16] and see also the current ICSO proceedings [23] and [24] on the SPEXone instrument design and performance.

\section{SPEX AIRBORNE INSTRUMENT AND INSTRUMENT MODEL}

The optical module of SPEX airborne consists of nine identical pre-slit optical units -the polarization encoders- and a spectrometer. The interface between the two is an array of slits. The optical system, including several light paths in the plane of spectral dispersion, are shown in Figure 1. Nine identical units with pre-slit polarization modulation optics (PMO) are responsible for encoding the linear degree of polarization of the incoming light. Light passes the polarization encoder optics first, ensuring that no instrument polarization corrupts the signal before polarimetric encoding occurs. Two spectrally modulated beams exiting each of the nine polarization encoders are focused in pairs, by a $4.4 \mathrm{~mm}$ diameter lenslet with a focal length of $10 \mathrm{~mm}$, via a folding mirror onto a pair of slits of an array of eighteen identical slits. In the spectrometer, light is collimated via a spherical mirror, then folded via a flat mirror, and spectrally dispersed by a holographic grating. Light is subsequently re-focused by a set of a-spherical lenses onto a single CCD detector. The effective wavelength range of 400-760 is determined by the transmissive optics becoming opaque below 400nm, and by high order overlap from the grating at wavelengths above $760 \mathrm{~nm}$. A detailed description of the instrument is provided in [18] and [21].

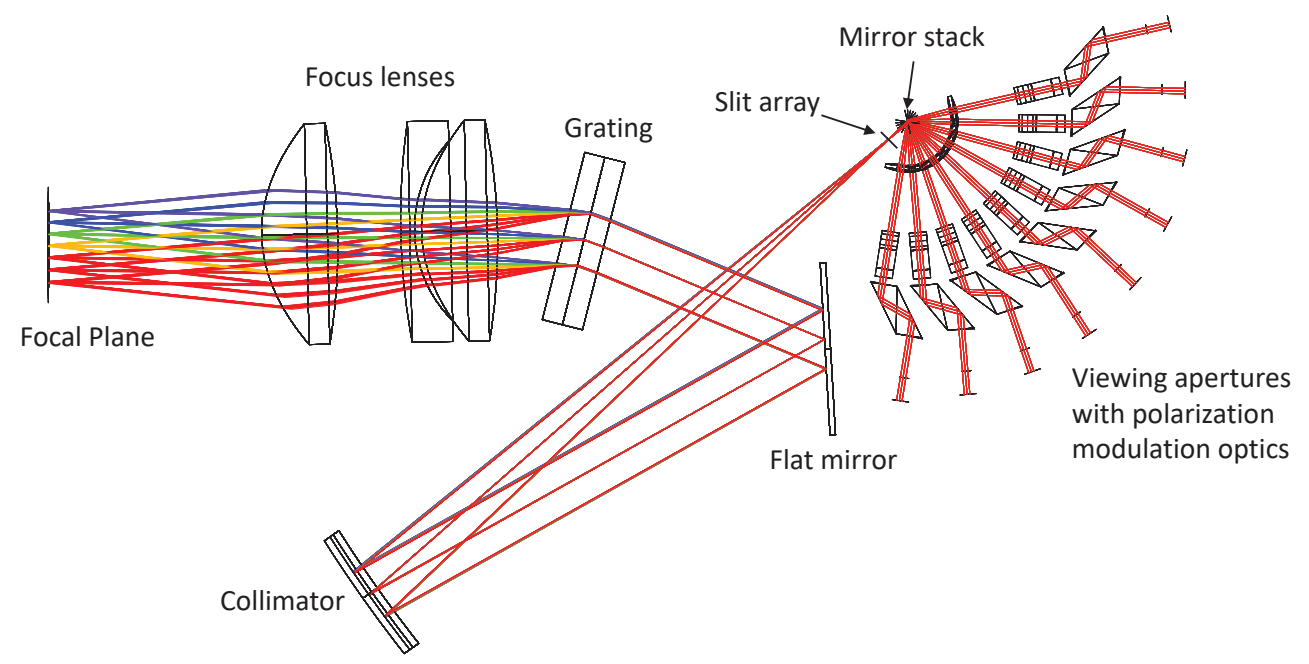

Figure 1. Representation of the OM optical components and light paths entering via the polarization modulation optics. The slit array is oriented normal to the paper. 


\subsection{Spectral Modulation}

Encoding of the state of linear polarization by the PMO occurs by a combination of three optical elements [8]: a quarter wave retarder, a (composite) multiple order retarder (MOR), and a polarizing beam-splitter (PBS). Light passing through the MOR undergoes a wavelength-dependent phase shift, resulting in a change of incident linearly polarized light along the vertical into circular, elliptical or linear polarization, as a function of wavelength. The PBS acts as two linear polarizers; the projection of the state of polarization onto each polarizer axis determines the intensity of the light passing through. After the PMO, two beams emerge, denoted as "S" and "P". The intensity spectrum of each beam is modulated sinusoidally, with the amplitude of the modulation proportional to the degree of linear polarization of the incident light:

$$
I_{S / P}(\lambda)=\frac{1}{2} I(\lambda)\left\{1 \pm p \cos \left(\frac{2 \pi \delta}{\lambda}+2 \beta\right)\right\} .
$$

Here $I(\lambda)$ is the incident spectral radiance, $p$ and $\beta$ are the degree and angle of linear polarization of the incident light respectively, $\lambda$ is the wavelength, $\delta$ is the retardance, and $I_{\mathrm{S}}(\lambda)$ and $I_{\mathrm{P}}(\lambda)$ are the two beams exiting the PMO. The retardance $\delta$ is $\sim 21 \mu \mathrm{m}$, resulting in a gradually increasing modulation period from $7 \mathrm{~nm}$ at $400 \mathrm{~nm}$ up to $28 \mathrm{~nm}$ at $760 \mathrm{~nm}$. The retardance is not quite constant but varies slightly with wavelength. The modulation amplitude scales with the degree of linear polarization $p$ (DoLP), while the phase $\beta$ equals angle of linear polarization (AoLP). The "+" and "“." in Eq.(1) apply to the S- and P-beam respectively. The two beams have orthogonal polarization, whereby their respective modulations are precisely in anti-phase. The function of the QWR is to change linearly polarized light with polarization axis along the optical axes of the MOR into circularly polarized light. One single parameter characterizes the instrument response, which is the retardance $\delta(\lambda)$. From Eq.(1) it follows that

$$
I(\lambda)=S_{S}(\lambda)+S_{P}(\lambda)
$$

i.e. the incident spectral radiance is readily obtained from the sum of $\mathrm{S}$ and $\mathrm{P}$ beam.

The angle and degree of linear polarization can be obtained from the modulation function

$$
F_{\text {mod }}(\lambda) \equiv \frac{S_{S}(\lambda)-S_{P}(\lambda)}{S_{S}(\lambda)+S_{P}(\lambda)}=p \cos \left(\frac{2 \pi \delta(\lambda)}{\lambda}+2 \beta\right)
$$

From the modulation function DoLP and AoLP can be retrieved by demodulation (Section 4), provided the retardance is known (from calibration). In the following sections several instrumental effects are considered leading to slight modification of the ideal expression Eq.(1).

\subsection{Mueller Matrix elements}

Equation (1) derives from a more general description of the optical system using Mueller matrix representation, see [21]:

$$
\vec{S}_{\text {out }}(\lambda)=\mathbf{M}(\lambda) \cdot \vec{S}_{\text {in }}(\lambda),
$$

where $\vec{S}=(I, Q, U, V)$ and $\mathbf{M}$ is a $4 \times 4$ matrix describing the instrument response. The Mueller matrix leading to Eq.(1) is

$$
\mathbf{M}_{\mathrm{PMO}}(k)=\frac{1}{2}\left(\begin{array}{cccc}
1 & k \cos \left(\frac{2 \pi \delta}{\lambda}\right) & -k \sin \left(\frac{2 \pi \delta}{\lambda}\right) & 0 \\
k & \cos \left(\frac{2 \pi \delta}{\lambda}\right) & -\sin \left(\frac{2 \pi \delta}{\lambda}\right) & 0 \\
0 & 0 & 0 & 0 \\
0 & 0 & 0 & 0
\end{array}\right), \quad k=+1,-1,
$$

where $=+1,-1$ applies to the S- and P- beam respectively. In our specific case we consider cases where circular polarization is absent, $V=0$ :

$$
\vec{S}_{\mathrm{in}}=I\left(\begin{array}{l}
1 \\
q \\
u \\
0
\end{array}\right)=I p\left(\begin{array}{c}
1 \\
\cos 2 \beta \\
\sin 2 \beta \\
0
\end{array}\right),
$$

in which $q=Q / I, u=U / I$. Note that $p=\sqrt{q^{2}+u^{2}}$ and $\beta=\frac{1}{2} \operatorname{atan}(u / q)$. The intensities of the S- and P- beams are obtained by inserting Eq.(5) and (6) in Eq.(4), and then considering the first element of the vector $\vec{S}_{\text {out }}$ :

$$
I_{s}(\lambda)=\frac{1}{2} I(\lambda)\left\{1+q \cos \left(\frac{2 \pi \delta}{\lambda}\right)-u \sin \left(\frac{2 \pi \delta}{\lambda}\right)\right\},
$$




$$
I_{P}(\lambda)=\frac{1}{2} I(\lambda)\left\{1-q \cos \left(\frac{2 \pi \delta}{\lambda}\right)+u \sin \left(\frac{2 \pi \delta}{\lambda}\right)\right\}
$$

which is identical to Eq.(1). In line with the Mueller matrix formulation, Eq.(1) can be written in a more general form:

$$
\begin{aligned}
& I_{S}(\lambda)=\frac{1}{2} I(\lambda)\left\{M_{S, 11}+q M_{S, 12}+u M_{S, 13}\right\}, \\
& I_{P}(\lambda)=\frac{1}{2} I(\lambda)\left\{M_{P, 11}+q M_{P, 12}+u M_{P, 13}\right\},
\end{aligned}
$$

All quantities in these two equations are wavelength dependent with the (current) exception of the $M_{11}$ matrix elements. For the ideal modulation scheme $\operatorname{Eq}(1), \quad M_{S, 11}=M_{P, 11}=1$, but as we shall see in Section 3.1 this is equivalent to incorporating radiometric calibration. For the off-diagonal terms

$$
M_{S, 12}=-M_{P, 12}=\cos \left(\frac{2 \pi \delta}{\lambda}\right) ; M_{S, 13}=-M_{P, 13}=-\sin \left(\frac{2 \pi \delta}{\lambda}\right) .
$$

The anti-symmetry property $M_{S, 12}=-M_{S, 12}$ and $M_{S, 13}=-M_{S, 13}$ is a nice to have because it guarantees that we can construct modulation-free and a modulation-only quantities like in Eqs.(2) and (3). As we will see later on, adopting the more general form Eqs.(9) and (10) of the system, without assuming that Eq.(11) is valid, allows to incorporate instrumental effects in a natural way.

\subsection{Instrument Spectral Response Function}

When $\mathrm{S}$ and $\mathrm{P}$ beams pass the spectrometer, the predominant effect is the spectrometer slit function, or Instrument Spectral Response Function (ISRF) which results spectral smearing of a spectrum $F(\lambda)$ incident on the slit:

$$
\langle F\rangle=\int_{-\infty}^{+\infty} d \lambda^{\prime} h\left(\lambda^{\prime}\right) F\left(\lambda^{\prime}-\lambda\right)
$$

In the case of SPEX airborne, the slit function is more or less a smoothened top-hat with a width of $\sim 3 \mathrm{~nm}$. A basic assumption made in the spectral modulation measurement is that all spectral features have characteristic wavelengths longer than the ISRF effective spectral width, with the exception of the rapid modulations in $M_{12}$ and $M_{13}$. I.e., the spectral dependence of $I(\lambda), q(\lambda)$ and $u(\lambda)$ is assumed to be "slow". Then convolution of the slit function with Eqs.(7) and (8) results in

$$
\begin{aligned}
& \left\langle I_{s}\right\rangle(\lambda)=\frac{1}{2} I(\lambda)\left\{1+q\left\langle\cos \left(\frac{2 \pi \delta}{\lambda}\right)\right\rangle-u\left\langle\sin \left(\frac{2 \pi \delta}{\lambda}\right)\right\rangle\right\}, \\
& \left\langle I_{P}\right\rangle(\lambda)=\frac{1}{2} I(\lambda)\left\{1-q\left\langle\cos \left(\frac{2 \pi \delta}{\lambda}\right)\right\rangle+u\left\langle\sin \left(\frac{2 \pi \delta}{\lambda}\right)\right\rangle\right\},
\end{aligned}
$$

where we have used $\langle I\rangle=I,\langle q\rangle=q,\langle u\rangle=u$. Since SPEX airborne is collecting reflected solar light, radiance is solar spectrum-like, or will have solar and atmospheric absorption features. In that case $\langle I\rangle \neq I$. This is ignored for the moment but will be addressed in Section 5. Averaging of the sine and cosine terms with the ISRF effectively reduces their amplitude. Because the modulation period in the wavelength domain decreases towards shorter wavelength, the effect of the ISRF is stronger at short wavelengths, reducing the modulation amplitude more than at longer wavelengths. This can be accounted for by adopting the following expressions:

$$
\begin{aligned}
& \left\langle I_{s}\right\rangle(\lambda)=\frac{1}{2} I(\lambda)\left\{1+W(\lambda)\left(q \cos \left(\frac{2 \pi \delta}{\lambda}\right)-u \sin \left(\frac{2 \pi \delta}{\lambda}\right)\right)\right\}, \\
& \left\langle I_{P}\right\rangle(\lambda)=\frac{1}{2} I(\lambda)\left\{1-W(\lambda)\left(q \cos \left(\frac{2 \pi \delta}{\lambda}\right)-u \sin \left(\frac{2 \pi \delta}{\lambda}\right)\right)\right\} .
\end{aligned}
$$

The function $W(\lambda)$ is denoted as the modulation amplitude, is s wavelength much more slowly than the modulations. So now we have the two (wavelength dependent) parameters $W(\lambda)$ and $\delta(\lambda)$ that describe the instrument response.

There are other factors which may result in additional deviations from the nominal modulation form Eq.(1). The spectrometer optics introduce deformations like keystone and spectral smile, which result in changes in the orientation of the slit image focal plane plus variations in point spread. Effectively this causes differences in the ISRF for S- and Pbeams, so that the convolutions in Eqs.(15) and (16) are actually different. This breaks the S-P anti-symmetry; $W_{S} \neq W_{P}$, i.e. the sum Eq.(5) is not valid. In addition misalignment of the modulation optics may introduce phase shifts between the $\mathrm{S}$ and $\mathrm{P}$ beams. In SPEX airborne, the quarter wave retarder (a Fresnel Rhomb) does not invoke a perfect $\pi / 2$ phase shift over the full wavelength range. Consequently, $u$-polarized light will be somewhat less polarized than $u$-polarized light. Finally, the assumption that we can write $\left\langle\cos \left(\frac{2 \pi \delta}{\lambda}\right)\right\rangle_{S}$ as $W_{S}(\lambda) \cos \left(\frac{2 \pi \delta}{\lambda}\right)$ (with only a single cosine term) is at least 
mathematically incorrect for a general ISRF. Then the assumption that the off-diagonal Mueller elements are simple cosines and sines is incorrect. Altogether, this leads to adopting the more general form Eqs.(9) and (10) for a description of the instrument response. In addition, we will consider possible transmission losses along the optical path in the spectrometer, down to the detector, where conversion to a digital signal occurs. Ultimately the signal at detector level is modelled as follows:

$$
\begin{aligned}
& C_{S}(\lambda)=\frac{1}{2} I g_{S}\left\{M_{S, 11}+q M_{S, 12}+u M_{S, 13}\right\}, \\
& C_{P}(\lambda)=\frac{1}{2} I_{P}\left\{M_{P, 11}+q M_{P, 12}+u M_{P, 13}\right\},
\end{aligned}
$$

where $g_{S}(\lambda)$ and $g_{P}(\lambda)$ are radiometric "gain" factors which may be different for both beams.

\section{CALIBRATION}

Two aspects of instrument calibration discussed here: radiometric and polarimetric calibration. Other aspects of SPEX instrument calibration are discussed in detail in [21]. Here, "calibration" means the process of obtaining instrumental parameters from calibration measurements.

\subsection{Radiometric calibration}

Radiometric calibration data are achieved by exposing the instrument to an unpolarized $(q=u=0)$ light source. We use a 30cm diameter CSTM-USLR-V12F integrating sphere from LabSphere, in combination with a QTH-150 150 Watt quartz-tungsten-halogen light source. The output of the integrating sphere is radiometrically calibrated (NIST-traceable) by the manufacturer, so that the radiance $I=I_{\text {ref }}(\lambda)$ is known to a high level of accuracy (to within 1-2\%). Then from the measured signals $C_{S}(\lambda)$ and $C_{P}(\lambda)$ the factors $g_{S} M_{S, 11}$ and $g_{P} M_{P, 11}$ are determined via the relation

$$
C_{S, \text { unpol }}(\lambda)=\frac{1}{2} I_{\text {ref }}(\lambda) g_{S} M_{S, 11} ; C_{P, \text { unpol }}(\lambda)=\frac{1}{2} I_{\text {ref }}(\lambda) g_{P} M_{P, 11}
$$

The factors $g_{S} M_{S, 11}$ and $g_{P} M_{P, 11}$ are subsequently used to convert recorded signals to quantities with dimensions of radiance,

$$
I_{S}(\lambda)=C_{s}(\lambda) /\left(g_{S} M_{S, 11}\right) ; I_{P}(\lambda)=C_{S}(\lambda) /\left(g_{S} M_{S, 11}\right)
$$

Inserting this in Eqs.(17) and (18), the result of the radiometric calibration is that we may write

$$
\begin{aligned}
& I_{S}(\lambda)=\frac{1}{2} I\left\{1+q m_{S, q}+u m_{S, u}\right\}, \\
& I_{P}(\lambda)=\frac{1}{2} I\left\{1+q m_{P, q}+u m_{P, u}\right\},
\end{aligned}
$$

where $m_{S, q}=M_{S, 12} /\left(g_{S} M_{S, 11}\right)$, and others terms likewise. This shows that after radiometric calibration there are four wavelength dependent parameters $m_{S, q}(\lambda), m_{S, u}(\lambda), m_{P, q}(\lambda)$ and $m_{P, u}(\lambda)$ describing the instrument response, which are to be determined in the next calibration step. In is noted that all four Mueller matrix elements have the ISRF imprint.

\subsection{Polarimetric Calibration}

Polarimetric calibration data, i.e. knowledge of the Mueller matrix elements $m_{S, q}(\lambda), m_{S, u}(\lambda), m_{P, q}(\lambda)$ and $m_{P, u}(\lambda)$ as a function of wavelength, is achieved by exposing the instrument to $100 \%$ polarized light. For SPEX airborne a wiregrid polarizer is used with a large extinction ratio $\left(>10^{4}\right)$, in combination with the unpolarized lightsource setup in the previous section. The instrument response is recorded when the polarizer is rotated from 0-360 degrees in discrete angular. To determine four values $m_{S, q}, m_{S, u}, m_{P, q}$ and $m_{P, u}$, four polarizer angles are in principle sufficient, but we typically take steps of 15 degrees for an accurate solution. With the polarizer set at an angle $\beta$, the input calibration signal is then given by

$$
\left\{I=t I_{\text {ref }}, q=\cos 2 \beta, u=\sin 2 \beta\right\} .
$$

Transmission of the polarizer is contained in $t(\lambda)$. The raw signals are then

$$
C_{S}(\lambda, \beta)=\frac{1}{2} t I_{\text {ref }}\left\{M_{S, 11}+\cos 2 \beta M_{S, 12}+\sin 2 \beta M_{S, 13}\right\} \text {. }
$$

For the P-beam, replace subscript $\mathrm{S}$ with $\mathrm{P}$. An example of the instrument response as a function of wavelength is displayed in Figure 2a for two of SPEX airborne's viewports, with S and P spectra extracted for one field of view element in the center of the swath. It is noted that while for the nadir viewport the S- and P- response appear in anti-phase when the polarizer is turned, this is not strictly the case for all viewports, as can be seen in the aftward viewport in in Figure 2c. 
At a fixed wavelength $\lambda$, the counts $C_{S}$ undergo a double period variation when the polarizer is turned over 360 degrees (Malus' law). Dividing by the unpolarized signals in Eq.(21) cancels out the light source:

$$
C_{S}(\lambda, \beta) / C_{S, \text { unpol }}(\lambda)=\frac{1}{2} t\left\{1+\cos 2 \beta m_{S, q}+\sin 2 \beta m_{S, u}\right\}
$$

To find $m_{S, q}$ and $m_{S, u}$, fit the measured ratio on the lhs of Eq.(27) with a fit function of the form

after which the Mueller matrix elements are obtained by

$$
f_{\text {fit }}(\beta)=M_{1}+\cos 2 \beta M_{2}+\sin 2 \beta M_{3}
$$

$$
m_{S, q}=\frac{M_{2}}{M_{1}} ; \quad m_{S, u}=\frac{M_{3}}{M_{1}}
$$

Repeating the procedure at all wavelengths of interest gives $m_{S, q}(\lambda)$ and $m_{S, u}(\lambda)$ as a function of wavelength (and likewise for the P-channel). The spectral behaviour of the Mueller matrix elements $m_{S, q}(\lambda)$ and $m_{P, q}(\lambda)$ is shown in the plots in the right in Figure 2. They are sinusoids with a wavelength dependent amplitude, Eqs.(15) and (16). Not shown are the Mueller elements $m_{S, u}(\lambda)$ and $m_{P, u}(\lambda)$. They are also sinusoids, and basically identical to $m_{S, q}(\lambda)$ and $m_{P, q}(\lambda)$ but shifted in phase by 90 degrees. In both graphs Figure $2 b$ and Figure $2 d$ it is evident that the modulation amplitude for $\mathrm{S}$ and $\mathrm{P}$ beams is not quite the same. This is shown more clearly in Figure 3, where the modulation efficiency is derived from the Mueller matrix elements:

$$
W_{S}(\lambda)=\sqrt{\left(m_{S, q}(\lambda)\right)^{2}+\left(m_{S, u}(\lambda)\right)^{2}},
$$

and likewise for the P-beam. For both viewports the modulation efficiency is smaller for the $\mathrm{P}$ beam than the $\mathrm{S}$ beam at the lower end of the spectrum. For the nadir viewport, the efficiencies converge towards each other at longer wavelengths, while for the aft viewport the $P$ beam becomes slightly more efficient above $600 \mathrm{~nm}$. Note also how overall the polarimetric efficiency of the aft viewport is much smaller at lower wavelengths than the nadir viewport. In Figure 3 the modulation efficiency drops sharply at wavelengths above $\sim 760 \mathrm{~nm}$. At longer wavelengths transmission ratio of the polarizer drops to low values, and the calibration measurements are invalid there. 

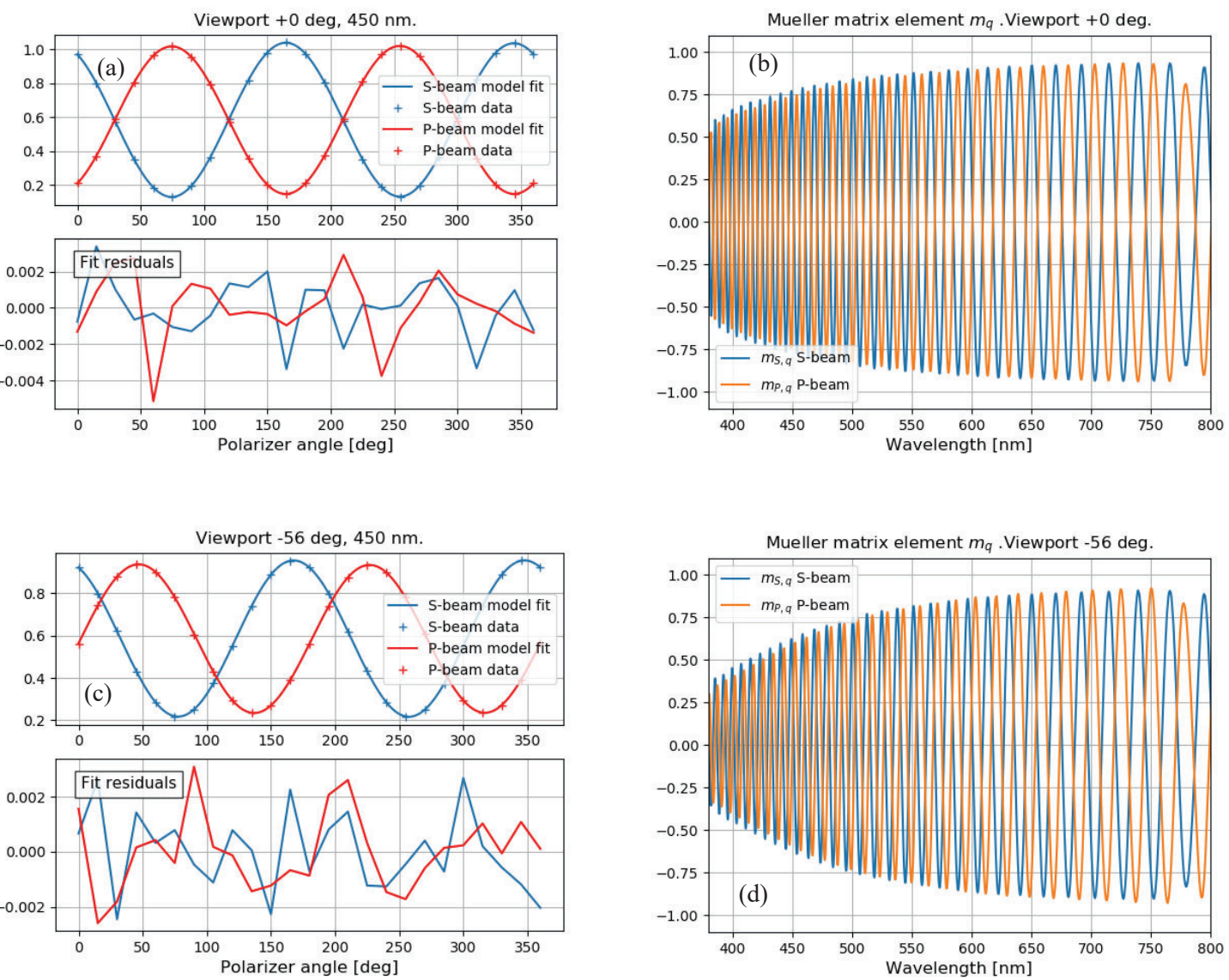

Figure 2. Left: Malus law, single wavelength fit. Right: derived Mueller elements $m_{S, q}$ and $m_{P, q}$. For the outermost viewing angle, there is a phase shift, due to a wavelength calibration error. It is of no concern however.
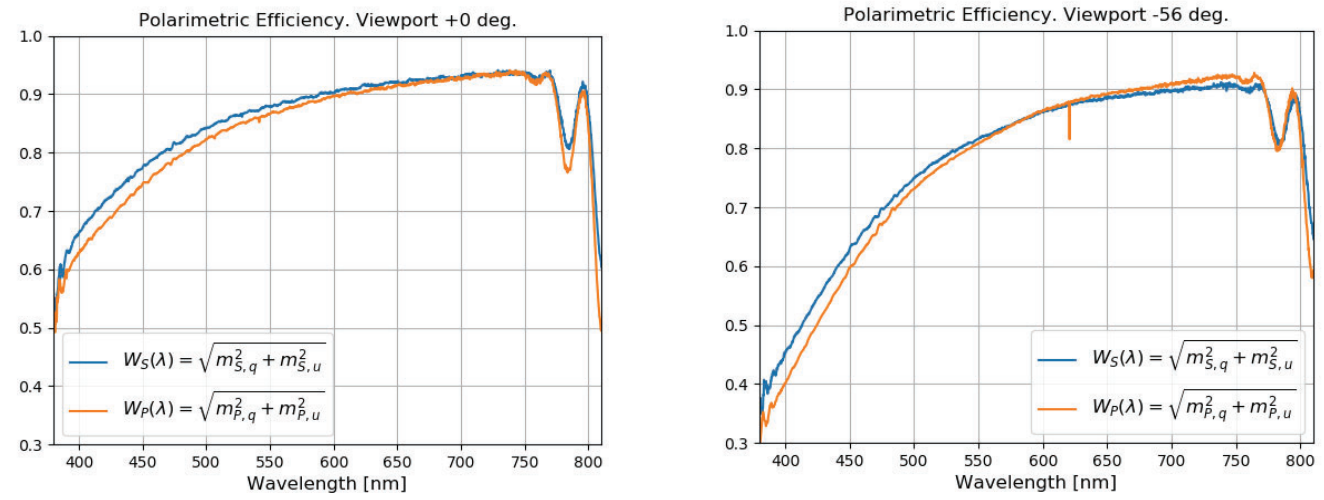

Figure 3. Modulation efficiencies derived from the Mueller elements for two viewports of SPEX airborne. Left: nadir viewport. Right: aftmost viewport looking "back" at an angle of 56 degrees. The right plot shows an anomaly in the form of a dip which is due to badly behaving pixels in the detector. 
It is noted that the lightsource $I_{\text {ref }}$ cancels in Eq.(25) only when it is sufficiently stable in time, or if the (spectrally) integrated output is recorded. In our case, the lightsource is typically stable to within $0.5 \%$ over the entire measurement, while also the integrated output is recorded with a silicon sensor integrated in the integrating sphere. An advantage of this approach is that it involves a linear fit-function. Moreover, prior radiometric calibration of $C_{S}(\lambda)$ is not required, since it would cancel in taking ratio $I_{S}(\lambda, \beta) / I_{S \text {, unpol }}(\lambda)$. Therefore possible radiometric calibration errors (or noise) don't affect the polarimetric calibration in this approach.

An alternative approach is to construct the modulation function Eq.(3) from the radiometrically corrected signals $I_{S}(\lambda)$ and $I_{S}(\lambda)$, and fitting the measurements as a function of polarizer angle $\beta$ with a fit-function (insert Eq.(23) and (24) in Eq.(3)):

$$
F_{\text {mod,fit }}(\beta)=\frac{\Delta m_{q} \cos 2 \beta+\Delta m_{u} \sin 2 \beta}{2+\Sigma m_{q} \cos 2 \beta+\Sigma m_{u} \sin 2 \beta}
$$

with fit four fit parameters

$$
\begin{aligned}
& \Delta m_{q}=m_{S, q}-m_{P, u} ; \Delta m_{u}=m_{S, u}-m_{P, u} \\
& \Sigma m_{q}=m_{S, q}+m_{P, q} ; \Sigma m_{u}=m_{S, u}+m_{P, u}
\end{aligned}
$$

In principle the separate Mueller elements can be derived from these fit parameters. Like in the former approach, fitting is performed separately at each wavelength. A slight disadvantage of this approach is that the fit-function is non-linear, requiring an iterative solver to find a solution. However, solutions are found rapidly for the initial guess S-P anti-symmetry is assumed, $\Sigma m_{q}=\Sigma m_{u}=0$, and then solve the resulting linear expression for $\Delta m_{q}$ and $\Delta m_{u}$. An advantage of this approach is that it is independent of the light source and variations thereof because $I_{\text {ref }}$ cancels in the construction of the modulation function.

\section{DEMODULATION}

Demodulation is the process of deriving the DoLP from recorded $\mathrm{S}$ and $\mathrm{P}$ spectra. From polarimetric calibration measurements (previous section), Mueller matrix elements have been obtained and are known functions of wavelength. Either in the form $m_{S / P, q}(\lambda), m_{S / P, q}(\lambda)$ or in the form $\Delta m_{S / P}(\lambda)$ and $\Sigma m_{S / P}(\lambda)$. To be precise, they are known at discrete wavelength values $\lambda_{i}$. In SPEX airborne, spectral sampling occurs at a spectral resolution of $\sim 1 \mathrm{~nm}$. The first step is to construct the modulation function from measured radiometrically spectra $I_{s}\left(\lambda_{i}\right)$ and $I_{P}\left(\lambda_{i}\right)$ :

$$
F_{\text {meas }, i}=\frac{I_{s}\left(\lambda_{i}\right)-I_{P}\left(\lambda_{i}\right)}{I_{s}\left(\lambda_{i}\right)+I_{P}\left(\lambda_{i}\right)}
$$

To derive the DoLP at a particular wavelength $\lambda_{0}$, take a number of number $N_{\lambda}$ of measurements $F_{\text {meas }}\left(\lambda_{i}\right)$ in an interval centered around $\lambda_{0}$, and fit the measurements $F_{\text {meas }, i}$ with a fit-function

$$
F_{\text {fit }}(\lambda)=\frac{\Delta m_{q}(\lambda) q_{0}+\Delta m_{u}(\lambda) u_{0}}{2+\Sigma m_{q}(\lambda) q_{0}+\Sigma m_{u, i}(\lambda) u_{0}}
$$

The solution $\left(q_{0}, u_{0}\right)$ is obtained from the over-determined set of equations

$$
F_{\text {meas }, i}-F_{\text {fit }}\left(\lambda_{i}\right)=0, \quad i=1, N_{\lambda} \text {. }
$$

For SPEX airborne, we typically take a spectral fit-window of one modulation period long, which ranges from $\sim 7 \mathrm{~nm}$ at $400 \mathrm{~nm}$ to $20 \mathrm{~nm}$ at $800 \mathrm{~nm}$, and 7 up to 10 equations to solve. For a rapid solution of Eq.(34) we start assuming S-P symmetry $\left(\Sigma m_{q}=\Sigma m_{u}=0\right)$ so that the system of equations is linear and can be solved in a single go. Next, the solution is taken as a starting point for solving the non-linear form iteratively. The DoLP then follows simply with $\mathrm{DoLP}=\sqrt{q_{0}^{2}+u_{0}^{2}}$. After demodulation, radiance can be obtained with

$$
I_{\text {meas }}\left(\lambda_{0}\right)=\frac{I_{\mathrm{S}}\left(\lambda_{0}\right)+I_{\mathrm{S}}\left(\lambda_{0}\right)}{1+\frac{1}{2} \Sigma m_{q}\left(\lambda_{0}\right) q_{0}+\frac{1}{2} \Sigma m_{u}\left(\lambda_{0}\right) u_{0}}
$$

in which $q_{0}$ and $u_{0}$ have been obtained from the demodulation step. Note again that in case of S-P anti-symmetry, this expression reduces to Eq.(3), so that the radiance can be determined independently from demodulation. A more refined solution may be constructed by adopting a parameterized dependence of the stokes elements, e.g. in the form of a polynomial

$$
q(\lambda)=\sum_{k=0}^{M} a_{k} \lambda^{k} ; u(\lambda)=\sum_{k=0}^{M} b_{k} \lambda^{k} \quad k=1, M .
$$

which are inserted in $\operatorname{Eq}(33)$ in place of $q_{0}$ and $u_{0}$. Naturally, the number of measurements $N_{\lambda}$ must be larger than the number $2 M$ of parameters. For SPEX airborne a linear spectral dependence is adopted when demodulating over one 
modulation period. Alternatively, one may extend the spectral range to the full spectrum. This can be an attractive approach when for example it is known that $q(\lambda)$ and $u(\lambda)$ are smooth functions of wavelength over the wavelength range of interest. Then a parameterized solution can be more accurate (less noisy) than a per-wavelength solution, because fewer parameters are fitted with the same number of measurements.
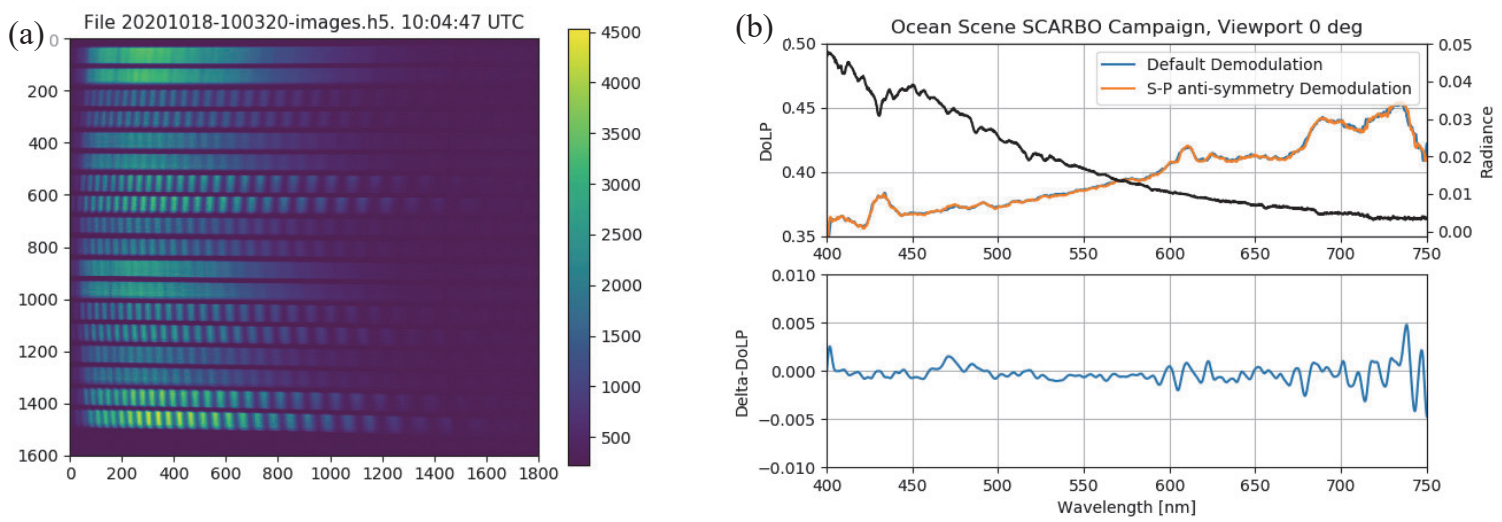

Figure 4. Left: raw detector image obtained during the SCARBO flight campaign in October 2020. Wavelength increases with detector colum, towards the right. For each of the nine viewports there are two spectra, S and P. Right: DoLP and radiance $I(\lambda)$ derived for the Nadir viewport. Black line is the spectral radiance $I(\lambda)$, DoLP is shown in blue and orange, where the blue line corresponds with the default solution obtained using Eq.(33), while the orange is obtained when adopting S-P symmetry.

An example of a single SPEX airborne measurement is show in Figure 4, recorded during the SCARBO flight campaign in October 2020 when the aircraft was flying over the Mediterranean sea, approaching Granada (Spain). The raw detector image in Figure 4(a) shows eighteen spectra aligned top down, where two adjacent spectra form an S and P pair. Already in the raw image spectral modulation is clearly present, with varying amounts of polarization in different viewing angles. Two of the viewing angles which show the least amount of polarization had land in sight, while all other viewports were viewing the sea. Figure 4(b) shows the spectral radiance radiance $I(\lambda)$ and $\operatorname{DoLP}(\lambda)$ as derived for the nadir viewport (the middle two spectra in Figure 4(a)). Radiance $I(\lambda)$ (black curve) shows atmospheric absorption features, while the DoLP is a much more smooth function of wavelength. The DoLP was derived taking two approaches. The "default" modulation, by solving Eq.(33) is the blue curve in Figure 9(b), while the orange curve was obtained by adopting S-P symmetry both in calibration and in demodulation. With this assumption we ignore differences in the polarimetric efficiency (Figure 3 ). The difference between the two approaches is displayed in the bottom graph of Figure 4(b). The two are in remarkable good agreement, with an rms difference of 0.002 over the range 400-760nm. For the other viewports the agreement between the two approaches is of the same order. This particular scene has rather high DoLP and represents an almost worst case test of the two methods, because we expect differences to increase with DoLP. In conclusion it seems that one may adopt $\mathrm{S}-\mathrm{P}$ anti symmetry even in the case when the differences in the polarimetric efficiency seem rather large. Apparently, if the S-P anti symmetrical approach is used consistently both in calibration and demodulation, the resulting error is second order (or higher) in the difference in modulation efficiencies of S and $\mathrm{P}$ beams.

\section{SPECTRAL FEATURES}

The recorded radiance spectrum in the previous section displays atmospheric absorption features. These features are in reality sharper than they appear Figure 4(b) because they have been smeared out by the ISRF. When the spectrum incident to SPEX has sharp spectral features, we expect errors in the derived DoLP that correlate with the spectral features. Figure 4(b) suggests that this occurs near 430nm, where a prominent "wiggle" in the DoLP spectrum appears to be correlated with a complex of lines in the solar radiance spectrum. In this section the effect of sharp spectral features on the DoLP is discussed. A remedy to deal with them is presented which requires knowledge of the instrument spectral response function (ISRF). For SPEX airborne this method has not been implemented in the data processing, because the required 
measurements to determine the ISRF are not available. Therefore the method is tested on simulated data. Before turning to the remedy, a simulation of the effect of sharp spectral features is shown in Figure 5. In this simulation, a simple model of the SPEX airborne instrument is constructed taking Eqs(1) as a starting point, using a 3nm wide top-hat slit function with gaussian edges to mimic point spread. Artificial calibration measurements are constructed with a spectrally smooth light source, from which the Mueller matrix elements are derived following the steps in 3.2. A simulated measurement is constructed using a full Stokes radiative transfer (Full Stokes) code (see, e.g. [22]) for the radiation field at the top of the atmosphere looking downward in different viewing directions. The DoLP is set to 0.6 (all wavelengths) and the AoLP is also fixed at an arbitrary value. The measurement is then simulated using the same instrument model, and the data are demodulated as in Section 4. Figure 5 shows the simulated radiation field and the DoLP. Clearly the derived DoLP deviates from the truth quite significantly, and the deviations correlate strongly with the spectral features of the incident radiation field.
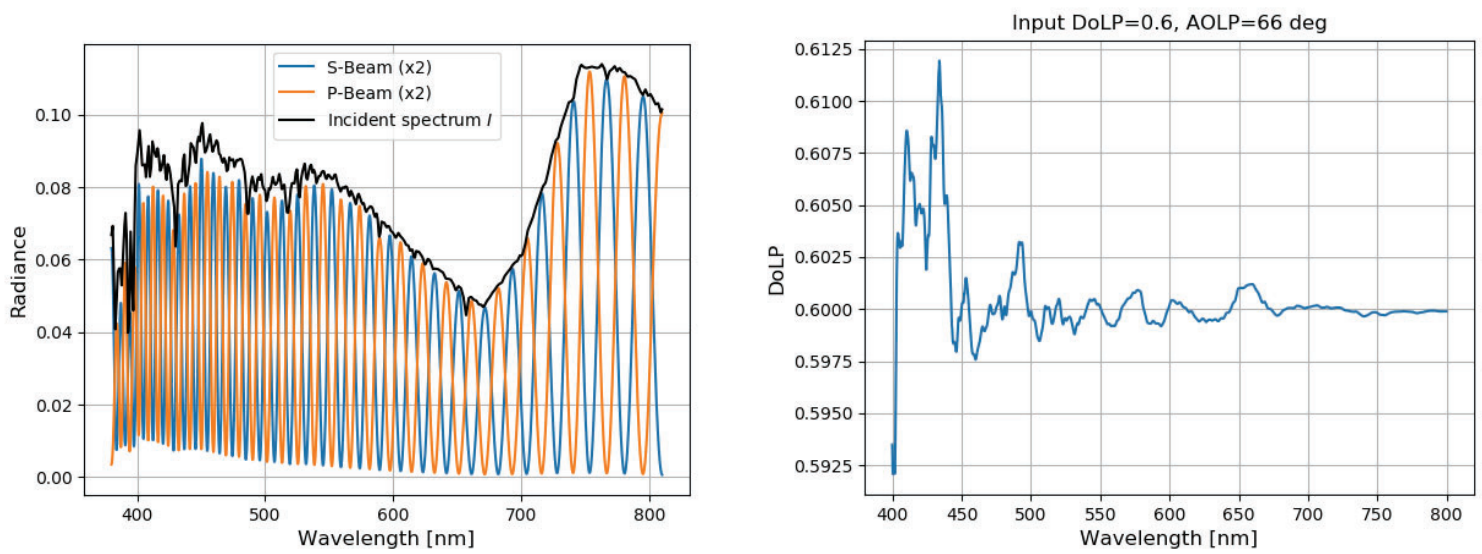

Figure 5. Simulated demodulation of a $100 \%$ polarized reflectance spectrum. Left: reflectance spectrum was calculated with a full-stokes radiative transfer code. Also shown are the simulated S- and P-beams. Right: DoLP after demodulation of the simulated data.

The simulations show that the spectrum to be recorded has sharp spectral features, with variations on a spectral scale shorter or comparable effective slit width, the basic assumption made in Section 2.3 that we can take out the radiance $I(\lambda)$ out from the convolution with the ISRF (Eqs.(13) and (14)) is no longer valid. We adopt here for simplicity S-P antisymmetry, but the discussion is valid also for the more general case. Also we expand only the expressions for the S-beam as the P-beam is readily obtained from the anti-symmetry. Starting with the modulation spectra incident on the spectrometer:

convolution with the ISRF leads to

$$
I_{S}(\lambda)=\frac{1}{2} I(\lambda)\left(1+m_{q}(\lambda) q(\lambda)+m_{u}(\lambda) u(\lambda)\right)
$$

$$
\left\langle I_{S}\right\rangle=\frac{1}{2}\left(\langle I\rangle+\left\langle I m_{q} q\right\rangle+\left\langle I m_{u} u\right\rangle\right)
$$

In polarimetric calibration measurements, light source was smooth, without prominent spectral features (In contrast to earlier calibration measurements. For a discussion on this, see [21]), so that from these measurements we have obtained $\left\langle m_{q}\right\rangle$ and $\left\langle m_{u}\right\rangle$, both of which do not feature in Eq.(38). A way out, or forward, is to make a less stringent assumption, by the notion that the 'rapid' spectral variations of $I$ (and also $Q$ and $U$ ) originate mostly from the rapid spectral variations in the incident solar flux $I_{\odot}(\lambda)$ at the top of the atmosphere, which has a fixed spectral shape, while spectral dependence of the normalized Stokes' parameters $q$ and $u$ is slow. We can then write

$$
I(\lambda)=f(\lambda) I_{\odot}(\lambda)
$$

where $f(\lambda)$ is some function with smooth spectral behaviour. Then we may write

$$
\langle I\rangle=f\left\langle I_{\odot}\right\rangle
$$

and also that $q$ and $u$ can be taken out of the convolution integral. For the modulation function we then have

$$
I \frac{\left\langle I_{S}\right\rangle-\left\langle I_{P}\right\rangle}{\left\langle I_{S}\right\rangle+\left\langle I_{P}\right\rangle}=\frac{q\left\langle I_{\odot} m_{q}\right\rangle+u\left\langle I_{\odot} m_{u}\right\rangle}{\left\langle I_{\odot}\right\rangle}
$$

So instead of calibration parameters $\left\langle m_{q}\right\rangle$ and $\left\langle m_{u}\right\rangle$ we should use modulation parameters 
For this to work, we must have knowledge of

$$
\frac{\left\langle I_{\odot} m_{12}\right\rangle}{\left\langle I_{\odot}\right\rangle} \text { and } \frac{\left\langle I_{\odot} m_{13}\right\rangle}{\left\langle I_{\odot}\right\rangle}
$$

- $\quad$ the instrument ISRF $h(\lambda)$

- $\quad$ the unconvolved functions $m_{12}(\lambda)$ and $m_{13}(\lambda)$

We should also account for the fact that the ISRF derived from instrument characterization/calibration measurements will not provide a perfect match with the actual ISRF of the instrument. The "true" ISRF determines the smoothing of Mueller matrix elements obtained from calibration. Therefore we introduce "revised" Mueller matrix elements

$$
\widetilde{m}_{q}(\lambda) \equiv \frac{\left\langle I_{\odot} m_{q}\right\rangle_{0}}{\left\langle I_{\odot}\right\rangle_{0}} \frac{\left\langle m_{q}\right\rangle}{\left\langle m_{q}\right\rangle_{0}}, \quad \tilde{m}_{u}(\lambda) \equiv \frac{\left\langle I_{\odot} m_{u}\right\rangle_{0}}{\left\langle I_{\odot}\right\rangle_{0}} \frac{\left\langle m_{u}\right\rangle}{\left\langle m_{u}\right\rangle_{0}}
$$

Where \langle\rangle$_{0}$ stands for convolution with an approximate slitfunction:

$$
\langle F\rangle_{0}=\int_{-\infty}^{+\infty} d \lambda^{\prime} h_{0}\left(\lambda-\lambda^{\prime}\right) F\left(\lambda^{\prime}\right)
$$

Such an approximate function may be derived for example from the optical model (ZEMAX Optics Studio), or from the characterization/calibration measurements. In the above equation, $\left\langle m_{q}\right\rangle$ and $\left\langle m_{u}\right\rangle$ are obtained from calibration of SPEX, in which the true ISRF determines the brackets. The idea is that the term $\frac{\left\langle I_{\odot} m_{q}\right\rangle_{0}}{\left\langle I_{\odot}\right\rangle_{0}}$ takes care of the rapid spectral variations in the solar spectrum, and that the term $\frac{\left\langle m_{q}\right\rangle}{\left\langle m_{q}\right\rangle_{0}}$ accounts for the fact that an approximate ISRF is used in $\frac{\left\langle I_{\odot} m_{q}\right\rangle_{0}}{\left\langle I_{\odot}\right\rangle_{0}}$. Convolutions $\left\langle m_{q}\right\rangle_{0}$ and $\left\langle m_{u}\right\rangle_{0}$ require knowledge from the "unconvolved" (or deconvolved) functions $m_{q}(\lambda)$ and $m_{u}(\lambda)$. Unconvolved functions $m_{q}(\lambda)$ and $m_{u}(\lambda)$ can for example be constructed from the retardance $\delta(\lambda)$ :

$$
m_{q}(\lambda)=\cos (2 \pi \delta / \lambda), m_{u}(\lambda)=-\sin (2 \pi \delta / \lambda)
$$

Our is that for SPEX airborne the retardance can be accurately derived from $\left\langle m_{q}\right\rangle$ and $\left\langle m_{u}\right\rangle$.
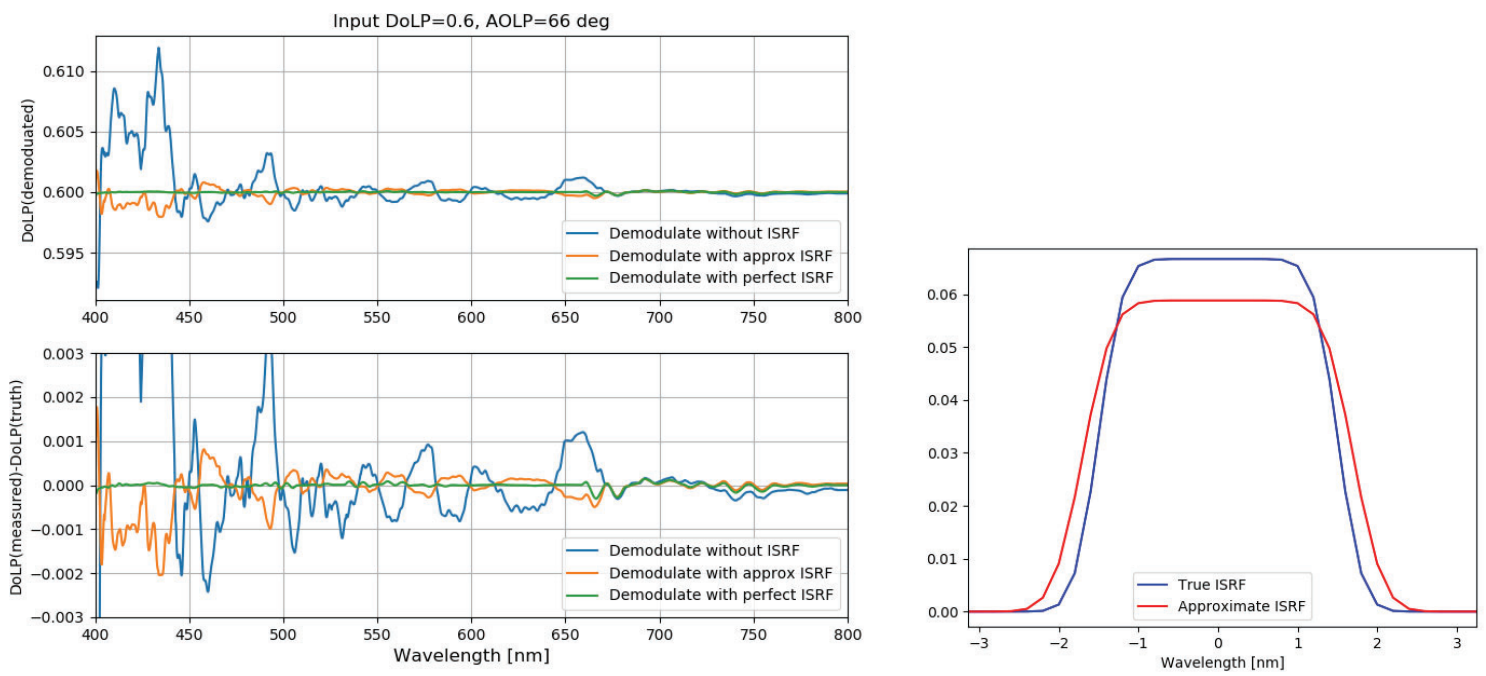

Figure 6. Left: Simulation of a DoLP measurement minus the truth (DoLP=0.6). The incident spectrum is the same as in Figure 5. Blue curve is the DoLP solution from gure 5, orange is the solutionusing $\widetilde{m}_{12}(\lambda)$ and $\widetilde{m}_{13}(\lambda)$ in the demodulation, and where the approximate ISRF differs significantly from the "true" ISRF. ISRF. True ISRF is a tophat of width $3 \mathrm{~nm}$, folded with a gaussian pointspread function with $\sigma=0.25 \mathrm{~nm}$. The approximative ISRF is a $3.2 \mathrm{~nm}$ wide tophat, folded 
with $\sigma=0.3 \mathrm{~nm}$. The green line is the DoLP solution in case one has perfect knowledge of the ISRF. Right: simulated ISRFs, where blue is the "true" ISRF of the instrument and red is the approximate version.

A test of this approach with revised Mueller matrix elements is shown in Figure 6, where de delta between measured DoLP and the truth are shown. In this simulation, the true ISRF has a slit width of $2 \mathrm{~nm}$ with a Gaussian convolution parameter of $\sigma=0.25$. The approximate ISRF is very different, with a slit width of $2.5 \mathrm{~nm}$ and $\sigma=0.3$. See the right graph in Figure 6 , Despite the fact that the ISRF is rather poorly estimated, the resulting DoLP error is 0.002 at most and only near the sharpest spectral features. With increased knowledge of the ISRF, the resulting DoLP measurement improves. The green curve in Figure 6 corresponds with the ideal case of perfect knowledge of the ISRF. The DoLP measurement, despite its negligible error here, is not perfect, and further improvement can only be obtained by a narrower ISRF.

\section{TELESCOPE-INDUCED POLARIZATION}

As explained in Section 1, the polarization optics in SPEXone are the first elements in the optical train. This way, there is no instrumental polarization contaminating the state of polarization prior to its encoding. Therefore we do not expect instrumental polarization to be a point of concern in SPEX airborne. In SPEXone on the other hand, the first optical element is a reflective three-mirror telescope, after which a PMO of a kind similar as in SPEXone encodes the polarization [16]. Here it is shown that telescope induced instrumental polarization (telescope polarization in short) poses no specific challenges when the Mueller matrix recipe is followed through. Telescope polarization can be described with:

$$
M_{t e l}=\left(\begin{array}{cccc}
1 & m_{t} & 0 & 0 \\
m_{t} & 1 & 0 & 0 \\
0 & 0 & 1 & 0 \\
0 & 0 & 0 & 1
\end{array}\right)
$$

For SPEXone, ZEMAX modelling indicates telescope polarization to be of order $m_{t} \sim 0.001-0.007$ [16]. With telescope polarization included, the Mueller matrix becomes

$$
\begin{aligned}
\boldsymbol{M}_{S / P}=\frac{1}{2} g_{s}\left(\begin{array}{ccccc}
1 & \pm \mathrm{m}_{\mathrm{q}} & \pm \mathrm{m}_{\mathrm{u}} & 0 \\
1 & \pm \mathrm{m}_{\mathrm{q}} & \pm \mathrm{m}_{\mathrm{u}} & 0 \\
0 & 0 & 0 & 0 \\
0 & 0 & 0 & 0
\end{array}\right) \cdot\left(\begin{array}{cccc}
1 & m_{t} & 0 & 0 \\
m_{t} & 1 & 0 & 0 \\
0 & 0 & 1 & 0 \\
0 & 0 & 0 & 1
\end{array}\right) \\
\quad=\frac{1}{2} g_{s}\left(\begin{array}{cccc}
1 \pm m_{t} m_{q} & m_{t} \pm m_{q} & m_{u} & 0 \\
1 \pm m_{t} m_{q} & m_{t} \pm m_{q} & m_{u} & 0 \\
0 & 0 & 0 & 0 \\
0 & 0 & 0 & 0
\end{array}\right)
\end{aligned}
$$

So that the S- and P- beam signals are given by

$$
\begin{aligned}
& C_{s}=\frac{1}{2} g_{s} I\left(\left(1+m_{t} m_{q}\right)+\left(m_{t}+m_{q}\right) q+m_{u} u\right) \\
& C_{p}=\frac{1}{2} g_{P} I\left(\left(1-m_{t} m_{q}\right)+\left(m_{t}-m_{q}\right) q-m_{u} u\right)
\end{aligned}
$$

which is of the same form at Eqs.(17)-(18). In this case however, the Mueller matrix elements $M_{11}$ are not constant. This means that the recorded signals $C_{s}$ and $C_{p}$ will display (small) residual spectral modulations even for unpolarized light $(q=u=0)$ due to the presence of $m_{q}$ in $M_{11}$. This is however of no concern. When performing radiometric calibration measurements as in Section 3.1, the factors $g_{\mathrm{s}}\left(1+m_{t} m_{q}\right)$ and $g_{\mathrm{s}}\left(1-m_{t} m_{q}\right)$ are determined. As before, the radiometric correction of $C_{s}$ and $C_{S}$ involves a division by these quantities:

$$
I_{S}=\frac{C_{S}}{g_{S}\left(1+m_{t} m_{q}\right)} ; I_{P}=\frac{C_{P}}{g_{P}\left(1-m_{t} m_{q}\right)}
$$

so that for the radiometrically corrected signals we have

$$
\begin{aligned}
& I_{s}=\frac{1}{2} I\left\{1+\frac{\left(m_{t}+m_{q}\right)}{\left(1+m_{t} m_{q}\right)} q+\frac{m_{u}}{\left(1+m_{t} m_{q}\right)} u\right\}, \\
& I_{P}=\frac{1}{2} I\left\{1+\frac{\left(m_{t}-m_{q}\right)}{\left(1-m_{t} m_{q}\right)} q-\frac{m_{u}}{\left(1-m_{t} m_{q}\right)} u\right\},
\end{aligned}
$$


Comparing with Eq.(21) and (22), we see that there is essentially no difference with the situation whithout telescope polarization. From polarimetric calibration as in Section 3.2 we obtain the elements (as a function of wavelength):

$$
\begin{aligned}
& \hat{m}_{S, q}=\frac{\left(m_{t}+m_{q}\right)}{\left(1+m_{t} m_{q}\right)} ; \hat{m}_{S, u}=\frac{m_{u}}{\left(1+m_{t} m_{q}\right)} \\
& \widehat{m}_{P, q}=\frac{\left(m_{t}-m_{q}\right)}{\left(1-m_{t} m_{q}\right)} ; \hat{m}_{P, u}=\frac{-m_{u}}{\left(1-m_{t} m_{q}\right)}
\end{aligned}
$$

This clearly shows that telescope polarization breaks the S-P anti-symmetry. From Eqs.(50) and (51) it is easy to derive $m_{q}, m_{u}$ and $m_{t}$ :

$$
\begin{gathered}
\left(m_{t} m_{q}\right)=\frac{\widehat{m}_{S, u}+\widehat{m}_{P, u}}{\widehat{m}_{S, u}-\widehat{m}_{P, u}}, \\
m_{u}=\left(1+\left(m_{t} m_{q}\right)\right) \widehat{m}_{S, u}, \\
m_{t}=\frac{1}{2}\left(\widehat{m}_{S, q}+\widehat{m}_{P, q}\right)+\frac{1}{2}\left(m_{t} m_{q}\right)\left(\widehat{m}_{S, q}-\widehat{m}_{P, q}\right), \\
m_{q}=\frac{1}{2}\left(\widehat{m}_{S, q}-\widehat{m}_{P, q}\right)+\frac{1}{2}\left(m_{t} m_{q}\right)\left(\widehat{m}_{S, q}+\widehat{m}_{P, q}\right),
\end{gathered}
$$

It is noted that these relations may not work in practice because in the denominator Eq.(52) has many zeropoints. For small $m_{t}$, a robust estimate is

$$
m_{t} \approx \frac{1}{2}\left(\widehat{m}_{S, q}+\widehat{m}_{P, q}\right)
$$

However, there is no strict need to perform the extraction of $m_{q}, m_{u}$ and $m_{t}$. Knowledge of elements $\widehat{m}_{S, q} \widehat{m}_{S, u}, \widehat{m}_{P, q}$ and $\widehat{m}_{P, u}$, is sufficient to perform the demodulation on the measured S- and P-beams to derive the DoLP. From the measured spectra $I_{S}(\lambda)$ and $I_{P}(\lambda)$ we can construct the (modulation-free) radiance by (c.f. Eq.(35) ):

$$
I=\frac{I_{S}+I_{P}}{2+\left(\widehat{m}_{S, q}+\widehat{m}_{P, q}\right) q+\left(\widehat{m}_{S, u}+\widehat{m}_{P, u}\right) u},
$$

The "proof" that indeed the Mueller approach works as it should with telescope polarization is shown in Figure 7 of a simulated SPEX measurement. The instrument model is the same as in the previous section, with the same ISRF and model parameters, but now telescope polarization is included in the Mueller matrix description of the instrument, and subsequently in the simulation of polarimetric and radiometric calibration measurements. A white radiance spectrum was used as input spectrum, with the DoLP varied from unpolarized to fully polarized. Figure 7 shows the measured DoLP error after demodulation with the blue curve labeled as "Default". The error is non-zero, but insignificant. Also in this figure is shown the case where S-P anti-symmetry is adopted in the polarimetric calibration and demodulation (orange curve). The behaviour of the DoLP error in this case is somewhat remarkable in the sense that the error is largest for unpolarized light $\mathrm{DoLP}=0$, and decreases with increasing DoLP with no error induced when the incident light is $100 \%$ polarized. 


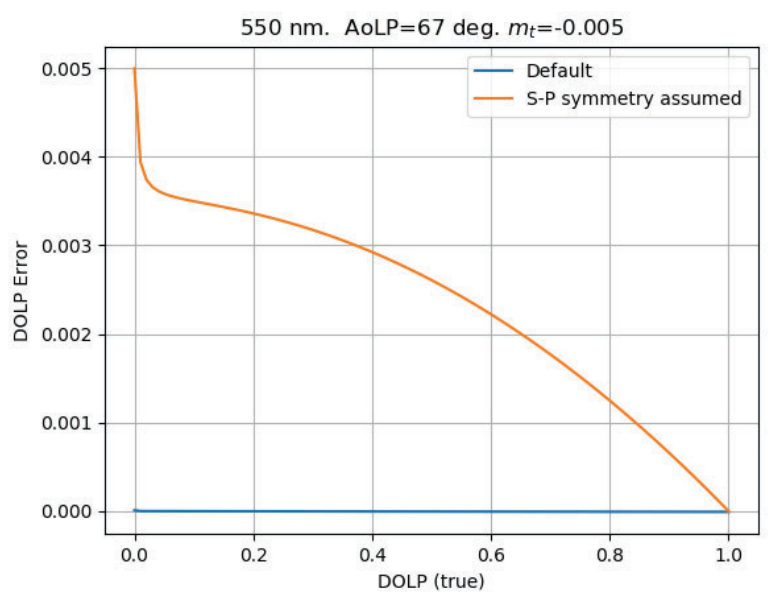

Figure 7. DoLP determined from input signal with range in DoLP (x-axis) and fixed AoLP $=67^{\circ}$, telescope polarization $m_{t}=-0.005$

\section{CONCLUSIONS AND OUTLOOK}

The present-day standard for remote sensing multi-angle polarimetric instrumentation for atmospheric aerosol characterization is to deliver polarimetric measurements with an error on the DoLP not larger than a few times 0.001. For SPEXone the requirement on the DoLP error is $<0.003$. Accurate polarimetric calibration is key to providing high-accurate polarimetric multi-angular measurements to derive atmospheric aerosol parameters. Here we have presented a procedure of polarimetric calibration and operational use for dual-beam SPEX airborne instrument following a Mueller matrix formulism of the instrument behaviour. It was shown how the approach allows to make few assumptions about the instrumental parameters, and is by construction tolerant for instrumental imperfections due to, e.g., optical distortions in the focal plane, mismatches in the two beams, and also possible instrumental polarization preceding the polarization encoding optics. A procedure for dealing with sharp features in the solar spectrum is presented which is shown to work well provided sufficient knowledge can be obtained of the instrument spectral response function. The lessons learned from SPEX airborne have been fed into the development of SPEXone and will also be employed in its calibration and data processing algorithms. A paper discussing polarimetric calibration of SPEXone and verified performance using a variable polarimetric stimulus is forthcoming. At the time of writing this contribution to the ICSO conference, SPEXone is being shipped for transport to NASA, where it will be integrated on the PACE spacecraft and launched in 2023.

\section{REFERENCES}

[1] Hasekamp O.P., Litvinov P., Butz A., Aerosol properties over the ocean from PARASOL multiangle photopolarimetric measurements, J. Geophys. Res., Vol. 116, D14204, doi:10.1029/2010JD015469 (2011)

[2] Dubovik O. et al., Statistically optimized inversion algorithm for enhanced retrieval of aerosol properties from spectral multi-angle polarimetric satellite observations, Atmos. Meas. Tech. 4, 975-1018 (2011)

[3] Lacagnina et al, Aerosol single-scattering albedo over the global oceans: Comparing PARASOL retrievals with AERONET, OMI, and AeroCom models estimates, J. Geophys. Res. Atmos., 120, 9814-9836, doi:10.1002/2015JD023501 (2015)

[4] Lacagnina C., Hasekamp O.P., Torres, O., Direct radiative effect of aerosols based on PARASOL and OMI satellite observations, J. Geophys. Res. Atmos., 122, doi:10.1002/2016JD025706 (2017) 
[5] Russel et al. A multiparameter aerosol classification method and its application to retrievals from spaceborne polarimetry, J. Geophys. Res. Atmos., 119, 98389863, doi:10.1002/2013JD021411 (2014)

[6] Erik Laan, Daphne Stam, Frans Snik, Theodora Karalidi, Christoph Keller, Rik ter Horst, Ramon Navarro, Gijsbert Oomen, Johan de Vries, Ruud Hoogeveen, "The Spectropolarimeter for Planetary Exploration: SPEX", Proc. SPIE 10566, International Conference on Space Optics - ICSO 2008, doi: 10.1117/12.2308240 (2008)

[7] Rietjens J.H.H. et al., SPEX: The Spectropolarimeter for Planetary Exploration, Proc. of the ICSO 2010, (2010)

[8] Snik, F., Rietjens, J. H. H., van Harten, G., Stam, D. M., Keller, C. U., Smit, J. M., Laan, E. C., Verlaan, A. L., ter Horst, R., Navarra, R., Wielinga, K., Moon, S. G. and Voors, R., "SPEX: The Spectropolarimeter for Planetary EXploration", Proc. SPIE 7731, 77311B (2010)

[9] Van Harten, G. et al., Prototyping for the Spectropolarimeter for Planetary EXploration (SPEX): calibration and sky measurements, Proceedings of SPIE 2011, Vol 8160 (2011)

[10] Rietjens, et al.. J.H.H.H et al., SPEX: a highly accurate spectropolarimeter for atmospheric aerosol characterization, Proceedings of ICSO (2014).

[11] Van Harten, G., Spectropolarimetry for Planetary Exploration, Ph.D. Thesis (2014)

[12] Rietjens J.H.H.R., Accurate spectrally modulating polarimeters for atmospheric aerosol characterization, Proceedings of SPIE, Volume 9613, (2015)

[13] Van Amerongen, A.H., et al, SPEX, the Dutch roadmap towards aerosol measurement from Space, Proceedings ICSO (2016).

[14] Van Amerongen, A.H., et al, SPEXone: A compact multi-angle spectro-polarimeter, Proceedings ICSO (2018).

[15] Snik F., Karalidi, T., Keller, C. U., Spectral modulation for full linear spectroscopy, Applied Optics 48, 1337 (2009)

[16] Jeroen Rietjens, Jochen Campo, Anantha Chanumolu, Martijn Smit, Raj Nalla, Cristina Fernandez, Jos Dingjan, Aaldert van Amerongen, Otto Hasekamp, "Expected performance and error analysis for SPEXone, a multi-angle channeled spectropolarimeter for the NASA PACE mission," Proc. SPIE 11132, Polarization Science and Remote Sensing IX, 1113208 (6 September 2019); https://doi.org/10.1117/12.2530729

[17] Van Harten, G. et al, Atmospheric aerosol characterization with a ground-based SPEX spectropolarimetric instrument, Atmos. Meas. Tech. Discuss., 7, 5741-5768, (2014)

[18] J. Martijn Smit, Jeroen H. H. Rietjens, Antonio di Noia, Otto P. Hasekamp, Wouter Laauwen, Brian Cairns, Bastiaan van Diedenhoven, A. Wasilewski, "In-flight validation of SPEX airborne spectro-polarimeter onboard NASA's research aircraft ER-2", Proc. SPIE 11180, International Conference on Space Optics — ICSO 2018, $111800 \mathrm{~N}$ (12 July 2019); https://doi.org/10.1117/12.2535942

[19] Europe eyes fleet of tiny CO2-monitoring satellites to track global emissions, Nature 562, 176-177 (2018) doi: https://doi.org/10.1038/d41586-018-06963-4

[20] Gousset, S., Croizé, L., Le Coarer, E. et al. NanoCarb hyperspectral sensor: on performance optimization and analysis for greenhouse gas monitoring from a constellation of small satellites. CEAS Space J 11, 507-524 (2019). https://doi.org/10.1007/s12567-019-00273-9

[21] J. Martijn Smit, Jeroen H. H. Rietjens, Gerard van Harten, Antonio Di Noia, Wouter Laauwen, Brian E. Rheingans, David J. Diner, Brian Cairns, Andrzej Wasilewski, Kirk D. Knobelspiesse, Richard Ferrare, and Otto P. Hasekamp, "SPEX airborne spectropolarimeter calibration and performance", Appl. Opt. 58, 5695-5719 (2019); https://doi.org/10.1364/AO.58.005695

[22] Hasekamp O. P., Landgraf J., Linearization of vector radiative transfer with respect to aerosol properties and its use in satellite remote sensing, JGR 110, D4, (2005), https://doi.org/10.1029/2004JD005260

[23] Jochen Campo, Paul Tol, Joris van der Vlugt, Martijn Smit, Jelle Talsma, Jens Johansen, Alexander Eigenraam, Richard van Hees, Otto Hasekamp, Marc Oort, Aaldert van Amerongen, and Jeroen Rietjens., Characterization and video chain development of the CMOS detector applied in the multi-angle spectro-polarimeter SPEXone, These Proceedings (2021)

[24] Jeroen Rietjens, Jochen Campo, Martijn Smit, Robbert Winkelman, Raj Nalla, Jochen Landgraf, Otto Hasekamp, Marc Oort, Aaldert van Amerongen, and SPEXone team, Optical and system performance of SPEXone, a multiangle channeled spectropolarimeter for the NASA PACE mission, These Proceedings (2021). 